Results 9,369 women returning to the same job within 1 year after delivery were included. Among them, 8,478 (90.5\%) mothers returned to work after no more than 2 months, when they would be assumed to have a full-paid maternity leave. Compared with those having stationary workload, those suffering from increased job stress during pregnancy had a 1.72 times greater risk of later RTW - between 6 and 12 months, after adjusting for potential confounders. Moreover, among those not returning to work more than 2 months after delivery, the aOR of later RTW was 2.0 (95\% CI: 1.25, 3.19) among those with increased job stress.

Conclusions In this prospective birth cohort study, mothers' prenatal work conditions were significantly related to later RTW for those returning to work within 1 year after childbirth.

\section{P-7 WHICH ARE THE DETERMINANTS INFLUENCING THE INTENSION OF NURSES TO STAY AT THEIR INSTITUTION}

${ }^{1}$ Miho Satoh. 'Yokohama City University, Japan

\subsection{6/OEM-2021-EPI.162}

Objective This study aimed to investigate the main determinants related to their intension to stay at an institution, by years of clinical experience, among nurses in Japan.

Method A cross-sectional survey was conducted at 12 hospitals in the Tohoku and Kanto regions of Japan. Of the 1,034 nurses working in those hospitals, 481 nurses (response rate: $46.5 \%$ ) completed a questionnaire and contributed to the analyses. The participants identified the determinants strengthening their intention to stay at the current hospital ('comfortable workplace environment,' 'passive motivational factors,' 'convenience of hospital location,' 'favorable worklife balance,' and 'fulfilment in nursing'), and individual attribution. The radar charts were shown to assess the determinants strengthening the intention to stay based on the standardized scores of determinants strengthening the intention to stay.

Results Nurses having less than 9 years of clinical experience and having 10-19 years of clinical experience showed higher scores of 'passive motivational factors' than the scores for the other determinants. Nurses having less than 9 years of clinical experience rated the lowest scores for 'favorable work-life balance' among all the determinants. On the other hand, nurses having more than 20 years of clinical experience gave the highest scores to 'favorable work-life balance', and the lowest scores to 'passive motivational factors.' Nurses having less than 9 years of clinical experience gave lower scores to 'comfortable workplace environment,' 'convenience of hospital location,' 'favorable work-life balance,' and 'fulfilment in nursing' than the other nurses. Nurses having more than 20 years of clinical experience showed opposite response trends compared to nurses having less than 9 years of clinical experience.

Conclusion Which determinants nurses emphasized in relation with their intention to stay would depend on the duration of clinical experience. Nurses having less than 9 years of clinical experience would be likely to stay at their current institution with passive motivation.

\section{P-9 THE COMBINED EFFECTS OF A HIGH PHYSICAL WORKLOAD AND EITHER OVERWEIGHT/OBESITY OR INSUFFICIENT VIGOROUS PHYSICAL ACTIVITY ON SELF- RATED HEALTH}

${ }^{1}$ Karin Proper, Mandy van den Berge, Sandra van Oostrom, Henk Van der Molen, Suzan Robroek, Carel Hulshof, Allard van der Beek. 'National Institute for Public Health and the Environment, Netherlands

\subsection{6/OEM-2021-EPI.163}

Introduction High physical workload and unhealthy lifestyle behaviors are common among blue-collar workers, both being separate risk factors for self-rated health. It may however be that the combination of high physical workload and an unhealthy lifestyle have a stronger effect than the sum of these factors, but studies to the combined effects are scarce. Objective To investigate the combined effects of a high physical workload and either overweight/obesity or insufficient vigorous physical activity on self-rated health.

Methods A longitudinal study was performed among 29,987 construction workers using data of two Workers\&rsquo; Health Surveillance Programs. Self-reported physical workload involved strenuous work postures and manual material handling. Insufficient vigorous physical activity was defined as self-reported vigorous activity for less than 3 times per week. Overweight/obesity was measured by physical examination. Self-rated health was measured using a single item question. Logistic regression analysis was used to investigate the associations between the separate risk factors at baseline and selfrated health at follow-up. The combined effects of physical workload and overweight/obesity or insufficient vigorous activity on self-rated health were analyzed using the relative excess risk due to interaction (RERI).

Results Construction workers with strenuous work postures (OR 1.35 95\%CI 1.25-1.46) or manual material handling (OR 1.29 95\%CI 1.19-1.40) were more likely to report poor self-rated health at follow-up. Overweight was not associated with poor self-rated health at follow-up, but obesity (OR 1.31 95\% CI 1.17-1.47) and insufficient vigorous activity (OR 1.13 95\%CI 1.01-1.25) were. However, no statistically significant interaction effects were found for physical workload and obesity or insufficient vigorous activity.

Conclusions Physical workload, obesity and insufficient vigorous activity were separate risk factors for poor self-rated health, but did not appear to have a synergistic effect. Worksite health promotion interventions focusing on improvement in physical workload and lifestyle both can have beneficial health effects.

\section{P-13 THE UTILITY OF OCCUPATIONAL HEALTH DATA IN THE CANADIAN PARTNERSHIP FOR TOMORROW'S HEALTH (CANPATH)}

${ }^{1}$ Ellen Sweeney, Philip Awadalla, Parveen Bhatti, Philippe Broet, Trevor Dummer, John McLaughlin, Donna Turner, Jennifer Vena. 'Dalhousie University, Canada

\subsection{6/OEM-2021-EPI.164}

Introduction The Canadian Partnership for Tomorrow's Health (CanPath) is a multi-centered prospective cohort study, and represents Canada's largest population health research 